\title{
Role of Fine Needle Aspiration Cytology And Histopathology in Diagnosis of Cervical Lymph Node Tuberculosis
}

\author{
Muniba Rahman ${ }^{1}$,Sajjad Ali Shahid ${ }^{2}$, Shahzad Ahmad $^{3}$, Muhammad Imran \\ Sarwar ${ }^{4}$, Muhammad Kashif Munir ${ }^{5}$, Sana Rehman $^{6}$,Iqra Riaz $^{7}$ \\ ${ }^{1,5,6,7}$ King Edward Medical University Lahore, ${ }^{2}$ The University Of Lahore, ${ }^{3}$ FMH College Of Medicine And \\ Dentistry, Lahore
}

\begin{abstract}
Background: Tuberculosis (TB) has been remained a major global health problem from ancient times. Diagnosis of $T B$ on the basis of histopathology is an important issue since long in anatomical pathology. Extra pulmonary $T B$ is predominantly associated with cervical lymph nodes presented by swollen glands usually at base and or sides of the neck. Taking limitations of serology, Ziehl Neelsen smear and culture techniques into the account morphological analysis based on histology seems to be the most achievable method for diagnosis of $T B$.

Objective: To see the value of Fine Needle Aspiration Cytology, Fine Needle Biopsy and or histopathology in diagnosis Cervical Lymph Node TB and to compare it with Acid Fast Bcilli smear and culture on Lowenstein Jensen medium.

Study Design \&Settings: This descriptive study was undertaken in Department of Pathology, King Edward Medical University, Lahore, in collaboration with all allied departments during January 2015 to December 2015.

Methods: After taking informed consent, patients were prepared for fine needle procedure under aseptic conditions. A senior pathologist took the specimen and then each specimen was divided into two parts. Hematoxylin and eosin staining was used to observe the morphological changes in the cells. Petroff's method of concentration and decontamination was used to process samples for ZN smear and culture.

Results: A total of 78 patients of age 15 years and above comprising of 21 males and 57 females with male to female ratio of 1:2.71 were included in present study. Mean age of the patients remained 26.55 \pm 14.09 . Out of total 78 patients $8(10.25 \%)$ provided surgically removed lymph node specimens, 6(7.69\%) provided small biopsies and 64 (82.05\%) were performed fine needle aspirate (FNA) for cytology. Sensitivity of AFB smear, culture and Histopathology/cytology findings remained $16.7 \%$, $89.7 \%$ and $93.6 \%$ respectively.

Conclusion:Histopathology/cytology is the only tool presenting timely morphological changes suggestive of Cervical Lymph Node TB and can be used to start anti tubercular treatment. Culture on Lowenstein Jensen medium, Ziehl Neelsen smear and response to anti tubercular treatment must not be ignored on the other hand and should be followed for proper management of disease.
\end{abstract}

Keywords: CLN, extra pulmonary TB, tuberculosis, ZN smear, LJ media, FNAC

\section{Introduction}

Tuberculosis (TB) has been remained a major global health problem from ancient times. Mycobacterium tuberculosis (MTB) is main culprit and cause of continuous morbidity and mortality. Mainly MTB attacks lungs and causes pulmonary tuberculosis however organism can infect every part of human body hence TB of any site other than lungs is termed as extra-pulmonary TB. Prevalence of Extra pulmonary TB ranges $19-30 \%$ in different in various studies ${ }^{1,2}$. Extra pulmonary TB is predominantly associated with cervical lymph nodes (CLN) presented by swollen glands usually at base and or sides of the neck $\mathrm{k}^{3}$.

Diagnosis of $\mathrm{TB}$ on the basis of histopathology is an important issue since long in anatomical pathology. Presence of granulomatous inflammation reaction around head and neck has been associated with wide range of idiopathic, immune, neoplastic, fungal and or infectious diseases ${ }^{4}$. Composition of granuloma is characterized by chronic inflammation caused by circulating monocytes in result of immune response. Histologically granulomatous reaction may be observed by transformed macrophages fenced by monocytes which later fused to show giant cells ${ }^{4}$. A thorough approach is desired to suggest a definite opinion because of wide variety diseases around neck.

Ziehl Neelsen (ZN) staining of acid fast bacilli (AFB) is recommended and method of choice for diagnosis of pulmonary TB in developing countries. Method is specific, smart and requires not more than two hours to produce smear results however sensitivity is as poor as less than $70 \%$ only ${ }^{5,6}$. In case of extrapulmonary $\mathrm{TB}$ the sensitivity of $\mathrm{ZN}$ smear is even lesser which may be reasoned paucibacillary nature of 
specimens, inadequacy, apportioning of specimen for various tests etc. resulting is uneven distribution of TB bacilli ${ }^{6}$. Serological tests on the other hand failed to impress the clinicians regarding diagnosis of TB. Culture for AFB on Lowenstein Jensen (LJ) media is still gold standard but the facility is not readily available, requires as long as six weeks to produce final results. Relatively faster, liquid culturesystems based on radiometric or non-radiometric mycobacterial growth indicator tests provide results in 2-3 weeks ${ }^{7}$ are costly, require high level infrastructure and more importantly need confirmation by making ZN smears again become more dangerous to play with live cultures.

Taking limitations of serology, $\mathrm{ZN}$ smear and culture techniques into the account morphological analysis based on histology seems to be the most achievable method for diagnosis of TB. Observation of granulomatous inflamed cells, foamy macrophages or Mycobacterial spindle cell pseudotumor are suggestive of TB and presence of TB bacilli need to be confirmed by smear or culture ${ }^{8}$. Polymerase chain reaction (PCR) based technique to diagnose TB fromtissue specimen has its own limitations and results are again only acceptable subject to the presence of beaded bacilli in the tissue ${ }^{9}$. Immunohistochemistry (IHC) is also an important technique though mentioned briefly in pathology books only hence does not provide standardized morphological criteria, therefore not well appreciated yet. Fine needle aspiration cytology (FNAC) and Fine needle biopsy (FNB) is relatively simple, safe, tolerated by patients easily and cost effective technique to get a clinical specimen from exact site of suspicion ${ }^{10}$.

Present study was undertaken to see the value of FNAC, FNB and or histopathology in diagnosis CLN TB and to compare it with AFB smear and culture on LJ medium.

\section{Materials \& Methods}

This descriptive study was undertaken in Department of Pathology and department of Chest Medicine, King Edward Medical University, Lahore, in collaboration with all allied departments who refer their patients for FNAC or send surgically removed lymph node samples for histopathology during January 2015 to December 2015. A semi structured questionnaire is used to collect the demographic information and history of the patients. Patients clinically suggestive for TB were termed as suspects and included in this study.

After taking informed consent, patients were prepared for FNB/FNAC of CLN under aseptic conditions. A senior pathologist took the specimen and then each specimen was divided into two parts. One half is immediately sent for ZN smear and culture and mycobacterial laboratory and other half to histopathology laboratory. Surgically removed tissue specimens were also divided in two portions and processed similarly as mentioned above. Tissue specimens received in formalinwere not included in the study as bacteriology of such specimens was not possible due to fixation.

Hematoxylin and eosin (H\&E) staining was used to observe the morphological changes in the cells and based on the principle that acid and basic component of the cells have affinity for basic and acidic dyes respectively. Hematoxylin being basic dye binds with acidic nucleus and eosin with basic cytoplasm. Histopathological smear shows bunching of immune cells in ring-a-ring-o-roses like appearance and multinucleated giant cell appearance due to fusion of many macrophages termed as granuloma. A dirty necrotic background suggests caseation may be due to TB.

Petroff's method of concentration and decontamination was used to process samples for ZN smear and culture $^{11}$. Smears were stained using $0.3 \%$ heated carbol fuchsin as primary stain, $3 \%$ acid alcohol as decolorizer and $0.3 \%$ methylene blue as secondary stain. Smears were observed under high power 100X oil immersion lens and results were reported according to criteria established by WHO/IUTALD (International union against tuberculosis and lung diseases) ${ }^{12}$. Cultures inoculated on LJ media were reported as described in another study from same settings ${ }^{6}$. Further patients were followed for ATT till the end of treatment and response to ATT was considered as gold standard in this study.

\section{Results}

A total of 78 patients of age 15 years and above comprising of 21 males and 57 females with male to female ratio of 1:2.71 were included in present study. Mean age of the patients remained 26.55 \pm 14.09 . Other demographic characteristics are shown in Table I. High number of patients 44/78 (56.4\%) were in elderly and younger age group of $15-24$ years and $48.7 \%$ of the total patients belong to poor socioeconomic status in this study. Previous history of treatment was present only $16.6 \%$ study subjects while history of contact and smoking were present in $66.7 \%$ and $21.8 \%$ patients respectively.

Out of total 78 patients $8(10.25 \%)$ provided surgically removed lymph node specimens, $6(7.69 \%)$ provided small biopsies and $64(82.05 \%)$ were performed fine needle aspirate (FNA) for cytology. Most of the suspects $62(79.48 \%)$ revealed granulomatous inflammation followed by 7(8.97) foamy macrophages, 4(5.13) spindle cells associated with Mycobacbacterial pseudo-tumors while 5(6.41) samples show no morphological changes. Type of specimens and histopathology/cytology findings are presented in table II. 
Culture on LJ medium and AFB smear were compared with histopathology/cytology findings and revealed $89.74 \%(70 / 78)$ very high as compared to AFB smear positivity which remained $16.67 \%$. Outcome of TB diagnosis culture remained significantly high ( $\mathrm{p}$-value <.05) as compared to AFB smear shown in table III.

Table I: Demographic Characteristics of Patients. ( $\mathrm{N}=78)$

\begin{tabular}{|c|c|c|c|c|}
\hline Sr. No. & Character & Description & $\mathbf{n}$ & $\%$ \\
\hline \multirow{2}{*}{1.} & \multirow{2}{*}{ Gender } & Male & 21 & 26.9 \\
\hline & & Female & 57 & 73.1 \\
\hline \multirow{2}{*}{2.} & \multirow{2}{*}{ Marital Status } & Married & 33 & 42.3 \\
\hline & & Unmarried & 45 & 57.7 \\
\hline \multirow{5}{*}{3.} & \multirow{5}{*}{$\begin{array}{l}\text { Age Groups } \\
\text { (In Years) }\end{array}$} & $15-24$ & 44 & 56.4 \\
\hline & & $25-34$ & 17 & 21.8 \\
\hline & & $35-44$ & 9 & 11.5 \\
\hline & & $45-54$ & 5 & 6.4 \\
\hline & & 55 and above & 3 & 3.8 \\
\hline \multirow{3}{*}{4.} & \multirow{3}{*}{ Socio-economic status } & Poor & 38 & 48.7 \\
\hline & & Middle & 25 & 32.0 \\
\hline & & High & 15 & 19.3 \\
\hline \multirow{2}{*}{5.} & \multirow{2}{*}{$\begin{array}{l}\text { Previous history of } \\
\text { treatment }\end{array}$} & Present & 13 & 16.6 \\
\hline & & Absent & 65 & 83.4 \\
\hline \multirow{2}{*}{6.} & \multirow{2}{*}{ History of Contact } & Present & 52 & 66.7 \\
\hline & & Not Established & 26 & 32.3 \\
\hline \multirow{2}{*}{7.} & \multirow{2}{*}{ History of smoking } & Present & 17 & 21.8 \\
\hline & & Absent & 61 & 78.2 \\
\hline \multirow{2}{*}{8.} & \multirow{2}{*}{$\begin{array}{l}\text { History of any other } \\
\text { Addiction }\end{array}$} & Present & 4 & 5.4 \\
\hline & & Absent & 74 & 94.6 \\
\hline
\end{tabular}

Table II: Type of Specimen and Histopathology/Cytology Findings. (N=78)

\begin{tabular}{|l|c|c|c|c|}
\hline \multirow{2}{*}{ Findings } & \multicolumn{3}{|c|}{ Type of specimen } & \multirow{2}{*}{$\begin{array}{c}\text { Total } \\
\text { n=78 }\end{array}$} \\
\cline { 2 - 4 } & $\begin{array}{c}\text { SRLN* } \\
\mathbf{n = 8}\end{array}$ & $\begin{array}{c}\text { Biopsy } \\
\mathbf{n = 6}\end{array}$ & $\begin{array}{c}\text { FNA } \\
\mathbf{n = 6 4}\end{array}$ & 62 \\
\hline Granulomatous Inflammation & 5 & 4 & 53 & 7 \\
\hline Foamy Macrophages & 1 & 1 & 5 & 4 \\
\hline Spindle Cells/Pseudo-tumors & - & 1 & 3 & 5 \\
\hline None & 2 & - & 3 & 78 \\
\hline Total & 8 & 6 & 64 & \\
\hline
\end{tabular}

*Surgically removed Lymph Node

Table III: Comparison of Histopathology/Cytology findings with AFB smear and Culture on LJ medium. $(\mathrm{N}=78)$

\begin{tabular}{|l|c|c|c|}
\hline Findings & Smear Positive & Culture Positive & p-value \\
\hline Granulomatous Inflammation & 10 & 58 & $<0.05$ \\
\hline Foamy Macrophages & 2 & 6 & $<0.05$ \\
\hline Spindle Cells/Pseudo-tumors & 1 & 4 & $<0.05$ \\
\hline None & - & 3 & $<0.05$ \\
\hline Total & 13 & 70 & $<0.05$ \\
\hline
\end{tabular}

Sensitivity of AFB smear, culture and Histopathology/cytology findings remained $16.7 \%, 89.7 \%$ and 93.6\% respectively taking response to ATT as gold standard while specificity of all three tests remained $100 \%$ in this study. Follow up of all the patients revealed relief from symptoms and complete loss of swollen lymph nodes at the end of treatment.

\section{Discussion}

Sensitivity of histopathology/cytology to diagnose the features suggestive of tuberculosis remained 93.6\% in present study was the highest compared to bacteriological techniques in current study and results are comparable with the study that presented sensitivity $91.3 \%$ for this technique furthermore morphological modifications of tissue by TB bacilli were demonstrated were of great importance ${ }^{8}$. Most of the cases $(82.05 \%)$ were performed FNA for cytology in current study may due to ease for both patient and laboratory staff. Utilization of FNAC has become the necessity of time for prompt investigation of lymphadenopathy and the technique is widely acceptable that requires minimum invasion with maximum output. Although a well-trained pathologist is compulsory to perform the procedure though the technique is rapid, simple, save and pain free relatively ${ }^{13}$.

Sensitivity of AFB smear by ZN staining remained $16.7 \%$ in the present study remained far low from another study done under similar settings but used wide range of extra-pulmonary and specimens which showed smear positivity of $35.7 \%{ }^{8}$. A varied range of $\mathrm{ZN}$ smear positivity of $0-75 \%$ has been reported in variety of 
studies reporting diagnosis of extra-pulmonary $\mathrm{TB}^{14}$. As this study included only CLN patients may be the reason of its lower sensitivity from other studies. Other factors related to low sensitivity of ZN smear may be the expertise of pathologist performing FNA. Required numbers of organisms for ZN stain to show a positive smear needs around 5000 bacilli per one milliliter of specimen. Perhaps smear positivity of FNA from CLN has been reported $25.1 \%$ and $49.7 \%$ in direct and concentrated specimen respectively in a recent study, thus minimized the attributing negative confounders ${ }^{15}$.

Response to ATT was considered as gold standard to calculate the sensitivity of all the techniques however culture on LJ medium is still gold standard for bacteriological detection of TB bacilli and its sensitivity remained $89.7 \%$ in this study. As the culture on LJ medium is time consuming and requires 6-8 weeks, therefore histopathology/cytology along clinical evaluation, history of ATT and history of contact are of abundant meaning to start ATT in CLN suspicious cases. Faster liquid cultures have shown their impact ${ }^{7}$ to replace culture on LJ media but could not establish in public sector due various factors like cost effectiveness, training of specialized staff and time to time maintenance and replacement of automatic systems. A sensitivity of $90 \%$ for culture on LJ medium has been reported by a previous study is in agreement with current study ${ }^{8}$.

Mean age of patients in current study remained $26.55 \pm 14.09$ with the fact that $56.4 \%$ CLN patients were in the age range of 15-24 years. This shows that our younger and adolescence age group is at higher risk of acquiring CLN. This study is comparable with the study that revealed $62.3 \%$ TB patients lie in most productive age group ${ }^{16}$. On the other hand male to female ratio of 1:2.71 in this study is alarming and not comparable at all with other studies ${ }^{16}$. A special focus on gender based studies is necessary to overcome the issue.

In conclusion, sensitivity of ZN smear for AFB is very low due to uneven dissemination of organism in the tissue and number of additional factors. Sensitivity of Culture on LJ is high enough but comprehensive delay in final results may badly affect the treatment outcome. Rapid molecular techniques like GeneXpert have limitations in processing of tissue specimens. In this situation histopathology/cytology is the only tool presenting timely morphological changes suggestive of CLN TB and can be used to start ATT. Culture on LJ medium, ZN smear and response to ATT must not be ignored on the other hand and should be followed for proper management of disease.

\section{References}

[1]. Kulchavenya E. Extrapulmonary tuberculosis: are statistical reports accurate?. Therapeu Advan Infect Dis. 2014;2(2):61-70.

[2]. Elmadhoun WM, Noor SK, Bushara SO, Ahmed EO, Mustafa H, Sulaiman AA et.al. Epidemiology of tuberculosis and evaluation of treatment outcomes in the national tuberculosis control programme, River Nile state, Sudan, 2011-2013. East Med Health J. 2016;22(2):95.

[3]. Tuberculosis. What is it? [Updated 2017, Cited 2017] accessed from URL: https://www.drugs.com/health-guide/tuberculosis.html

[4]. Granulomatous diseases of head and neck. [updated 2016, cited 2017] accessed from URL: http://emedicine.medscape.com/article/854739-overview

[5]. Hooja S, Pal N, Malhotra B, Goyal S, Kumar V, Vyas L. Comparison of Ziehl Neelsen \& Auramine O staining methods on direct and concentrated smears in clinical specimens. Indian J Tberc. 2011;58(2):72-6.

[6]. Munir MK, Anwar N, Iqbal R, Shabbir I, Nosheen S. Diagnosis of tuberculosis: molecular versus conventional method. Pak JMed Res. 2011;50(2):50-4.

[7]. Yam WC. Recent advances in rapid laboratory diagnosis of tuberculosis. Med Bull. 2006;11(1):6-7.

[8]. Karimi S, Shamaei M, Pourabdollah M, Sadr M, Karbasi M, Kiani A, Bahadori M. Histopathological findings in immunohistological staining of the granulomatous tissue reaction associated with tuberculosis. Tubercul Res Treat. 2014; http://dx.doi.org/10.1155/2014/858396

[9]. Ulrichs T, Lefmann M, Reich M, Morawietz L, Roth A, Brinkmann V et. al. Modified immunohistological staining allows detection of Ziehl-Neelsen-negative Mycobacterium tuberculosis organisms and their precise localization in human tissue. J Pathol. 2005;205(5):633-40.

[10]. Boon J. Biopsy, Fine Needle, Neck Mass. [Updated 2016, cited 2017] accessed from URL: http://emedicine.medscape.com/article/1520111overview?pa=eMnN5FK1W3M4ixN9ghtwaFEyBXEE\%2Fjr8ST9D9f2Ip5UXc2d48ppSMr8YTJH5F5i0auhyF\%2FhUra791wuSLzl BlaMMvXjUcyZ8uhFNFUtVAb4\%3D

[11]. Petroff's method for decontamination surveillance of anti-tuberculosis drug resistance. [cited 2017] accessed from URL: wwwn.cdc.gov/dls/ila/cd/.../Specimen\%20Decontamination.ppt

[12]. Tuberculosis control program. Implementing proficiency testing for TB smear microscopy in the Northern Province South Africa. 2001: 11-12. Available from: URL: http://www.hst.org.za/uploads/files/tbcontrol.pdf

[13]. Koo V, Lioe TF, Spence RA. Fine needle aspiration cytology (FNAC) in the diagnosis of granulomatous lymphadenitis. Ulster Med J. 2006;75(1):59-64.

[14]. Kamboj SN, Goel MM, Tandon PU, Natu SM, Nath P. Correlative study of histopathology and bacteriology in patients of tubercular lymphadenitis. Indian J Chest Diseases Allied Sci. 1993;36(4):187-91.

[15]. Tadesse M, Abebe G, Abdissa K, Bekele A, Bezabih M, Apers L et.al. Concentration of lymph node aspirate improves the sensitivity of acid fast smear microscopy for the diagnosis of tuberculous lymphadenitis in Jimma, southwest Ethiopia. PloS one. 2014;9(9):e106726.

[16]. Munir MK, Rehman S, Aasim M, Iqbal R, Saeed S. Comparison of Ziehl Neelsen Microscopy with GeneXpert for Detection of Mycobacterium Tuberculosis. IOSR-JDMS.;1(14):56-60. 